\title{
Corrigendum: Immune surveillance in the central nervous system
}

Shalina S Ousman \& Paul Kubes

Nat. Neurosci. 15, 1096-1101 (2012); published online 26 July 2012; corrected after print 10 February 2014

In the version of this article initially published, the figure that depicted the main sites of immune cell entry into the brain parenchyma contained some anatomical details that we felt might lead to confusion among the nonspecialist readership. The central region of the brain parenchyma was unlabeled and not colored as parenchyma, and the subarachnoid space that surrounds the cerebellum was not indicated. These omissions have been rectified. In addition, the folded epithelial cell layer lining the choroid plexus had been depicted projecting into the unlabeled central region. We now show this cell layer extending into the ventricle. The figure legend has also been updated to indicate that cells reach the CSF in the first route by crossing through the fenestrated blood vessels of the choroid plexus. The errors have been corrected in the HTML and PDF versions of the article.

\section{Addendum: Deep brain stimulation restores frontostriatal network activity in obsessive-compulsive disorder}

\begin{abstract}
Martijn Figee, Judy Luigjes, Ruud Smolders, Carlos-Eduardo Valencia-Alfonso, Guido van Wingen, Bart de Kwaasteniet, Mariska Mantione, Pieter Ooms, Pelle de Koning, Nienke Vulink, Nina Levar, Lukas Droge, Pepijn van den Munckhof, P Richard Schuurman, Aart Nederveen, Wim van den Brink, Ali Mazaheri, Matthijs Vink \& Damiaan Denys Nat. Neurosci. 16, 386-387 (2013); published online 24 February 2013; addendum published after print 18 July 2014

Reanalysis accounting for electrode artifacts. The newly published Supplementary Figure 6 depicts normalized EPI scans from two DBS-implanted subjects illustrating that the nucleus accumbens ROI (red) and the region of signal dropout around the electrode are not overlapping. Nevertheless, we reanalyzed our data to further rule out the possibility that our results were affected by signal measured from the dropout region. We re-analyzed the data by removing the parts of the ROI that would extend into the electrode dropout region based on the normalized but unsmoothed functional images instead of T1-weighted scans. We then extracted the ROI time series from these unsmoothed images and correlated these with the smoothed remaining brain. Results from this reanalysis (Supplementary Table 6 and Supplementary Fig. 7) are similar to those of the first analysis-that is, DBS-induced decrease in functional connectivity between the NAc and mPFC/ IPFC—confirming that our results are unlikely to reflect false positives related to electrode artifacts.
\end{abstract}

\section{Corrigendum: EZH2-mediated H3K27 trimethylation mediates} neurodegeneration in ataxia-telangiectasia

Jiali Li, Ronald P Hart, Elyse M Mallimo, Mavis R Swerdel, Alexander W Kusnecov \& Karl Herrup Nat. Neurosci. 16, 1745-1753 (2013); published online 27 October 2013; corrected after print 30 May 2014

In the version of this article initially published, red and blue were switched in the key and legend for Figure 3a. The error has been corrected in the HTML and PDF versions of the article.

\section{Corrigendum: Identification of a unique TGF- $\beta$-dependent molecular and functional signature in microglia}

Oleg Butovsky, Mark P Jedrychowski, Craig S Moore, Ron Cialic, Amanda J Lanser, Galina Gabriely, Thomas Koeglsperger, Ben Dake, Pauline M Wu, Camille E Doykan, Zain Fanek, LiPing Liu, Zhuoxun Chen, Jeffrey D Rothstein, Richard M Ransohoff, Steven P Gygi, Jack P Antel \& Howard L Weiner

Nat. Neurosci. 17, 131-143 (2014); published online 8 December 2013; corrected after print 19 December 2013

In the version of this article initially published, the $x$-axis labels for the sets of graphs in Figure $2 \mathrm{f}$ corresponding to astrocyte, oligodendrocyte and neuron molecules consisted of six items, even though there were only five bars. "Red pulp macrophages" was included in error. Also, the Cleveland Clinic affiliation gave the section as the Department of Immunology; the correct affiliation is Neuroinflammation Research Center. The errors have been corrected in the HTML and PDF versions of the article. 
Corrigendum: Post-study caffeine administration enhances memory consolidation in humans

Daniel Borota, Elizabeth Murray, Gizem Keceli, Allen Chang, Joseph M Watabe, Maria Ly, John P Toscano \& Michael A Yassa Nat. Neurosci. 17, 201-203; published online 12 January 2014; corrected online 17 January 2014; corrected after print 30 June 2014

In the version of this article initially published, there were errors in the reporting of statistics. In the Figure $1 \mathrm{~b}$ legend, the asterisked $P$ value was given in the HTML version as ${ }^{\star} P=0.05$ and in the $\mathrm{PDF}$ version as ${ }^{\star} P<0.05$. It should read ${ }^{\star} P<0.05$, one-tailed. In the Figure $2 \mathrm{a}$ legend, the degrees of freedom for the immediate caffeine group were given as 42 and the $P$ value as 0.05 ; the correct values are 71 and 0.049 , respectively. In the Figure $2 \mathrm{~b}$ legend and the fifth paragraph of the main text, the $P$ value for the main effect of caffeine was given as 0.001 ; the correct value is 0.05 . The errors have been corrected in the HTML and PDF versions of the article.

\section{Corrigendum: LRRK2 regulates synaptogenesis and dopamine receptor activation through modulation of PKA activity}

Loukia Parisiadou, Jia Yu, Carmelo Sgobio, Chengsong Xie, Guoxiang Liu, Lixin Sun, Xing-Long Gu, Xian Lin, Nicole A Crowley, David M Lovinger \& Huaibin Cai

Nat. Neurosci. 17, 367-376 (2014); published online 26 January 2014; corrected after print 27 February 2014

In the version of this article initially published, the G2385R mutation in Figure 7a,b was given as G2835R. The error has been corrected in the HTML and PDF versions of the article.

\section{Corrigendum: LRRK2 regulates synaptogenesis and dopamine receptor} activation through modulation of PKA activity

Loukia Parisiadou, Jia Yu, Carmelo Sgobio, Chengsong Xie, Guoxiang Liu, Lixin Sun, Xing-Long Gu, Xian Lin, Nicole A Crowley, David M Lovinger \& Huaibin Cai

Nat. Neurosci. 17, 367-376 (2014); published online 26 January 2014; corrected after print 27 February 2014; corrected after print 18 June 2014

Initial version

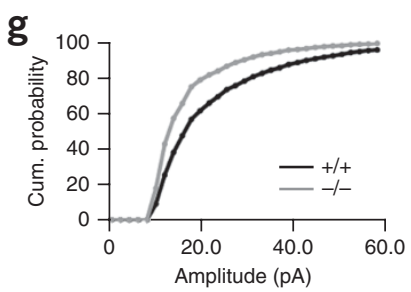

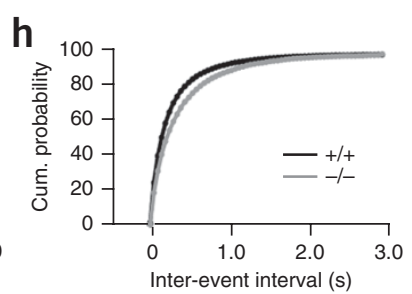

Corrected version
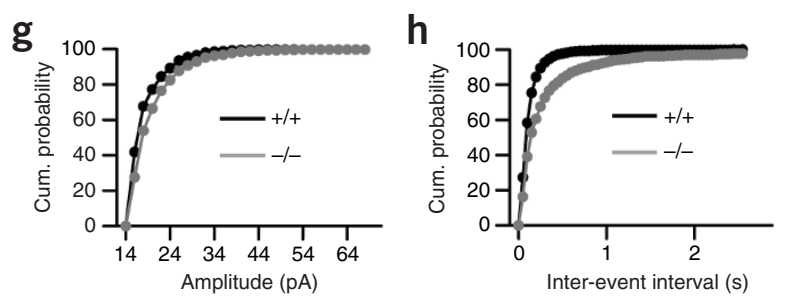

In the version of this article initially published, the line graphs presented in Figure 1g,h were switched with ones from a different experiment. The error has been corrected in the HTML and PDF versions of the article.

\section{Erratum: Peripheral gating of pain signals by endogenous lipid mediators}

Daniele Piomelli \& Oscar Sasso

Nat. Neurosci. 17, 164-174 (2014); published online 28 January 2014; corrected after print 18 March 2014

In the version of this article initially published, the phosphoanandamide in Figure 3a terminated in a hydroxyl instead of a phosphate group. The error has been corrected in the HTML and PDF versions of the article.

\section{Erratum: Orbitofrontal activation restores insight lost after cocaine use}

Federica Lucantonio, Yuji K Takahashi, Alexander F Hoffman, Chun Chang, Sheena Bali-Chaudhary, Yavin Shaham, Carl R Lupica \& Geoffrey Schoenbaum

Nat. Neurosci. 17, 1092-1099 (2014); published online 20 July 2014; corrected after print 28 July 2014

In the version of this article initially published, author Chun Yun Chang's name was given as Chun Chang. The error has been corrected in the HTML and PDF versions of the article. 